\title{
Melanoma Antigen Preferentially Expressed in Tumors
}

National Cancer Institute

\section{Source}

National Cancer Institute. Melanoma Antigen Preferentially Expressed in Tumors. NCI

Thesaurus. Code C127930.

Melanoma antigen preferentially expressed in tumors (509 aa, $\sim 58 \mathrm{kDa}$ ) is encoded by the human PRAME gene. This protein plays a role in the repression of retinoic acid receptor-dependent gene expression. 